\title{
Identification of the key genes associated with neuropathic pain
}

\author{
HUAN LIU ${ }^{1}$, TIANJIAO XIA ${ }^{1,2}$, FANGXIA XU ${ }^{1}$, ZHENGLIANG MA ${ }^{1}$ and XIAOPING GU ${ }^{1}$ \\ ${ }^{1}$ Department of Anesthesiology, Affiliated Nanjing Drum Tower Hospital of Nanjing University School of Medicine, Nanjing, \\ Jiangsu 210008; ${ }^{2}$ Jiangsu Key Laboratory of Molecular Medicine, Nanjing University School of Medicine, \\ Nanjing, Jiangsu 210093, P.R. China
}

Received October 8, 2017; Accepted February 9, 2018

DOI: $10.3892 / \mathrm{mmr} .2018 .8718$

\begin{abstract}
Neuropathic pain is a chronic pain state associated with multiple etiologies that results in considerable social and economic burden. The identification of key genes associated with neuropathic pain is important for the development of novel therapies. Therefore, the present study downloaded the gene expression profile GSE15041 from the Gene Expression Omnibus database. The unverified gene chip was removed and the microarray data was normalized following quality control. The limma package in $\mathrm{R}$ was used to screen the differentially expressed genes (DEGs), followed by Gene Ontology and Kyoto Encyclopedia of Genes and Genomes (KEGG) pathway enrichment analysis. Furthermore, a protein-protein interaction (PPI) network based on the identified DEGs was constructed to select hub proteins, and reverse transcription-quantitative polymerase chain reaction was performed to detect the expression of these proteins in a mouse model of neuropathic pain. In total, 86 common DEGs were identified. DEGs were significantly enriched in 'extracellular space' and KEGG pathway enrichment analysis demonstrated that the DEGs were significantly enriched in inflammatory diseases and the mitogen-activated protein kinase signaling pathway. The PPI network consisted of 27 nodes (proteins) and 47 PPI edges (interactions). Interleukin (IL)-6, transcription factor AP-1 (c-Jun) and urikinase-type plasminogen activator (Plau) were identified as hub proteins and key genes in neuropathic pain. The mRNA expression of these hub proteins was significantly increased in the neuropathic pain model, compared with the sham group. IL-6, c-Jun, and Plau may be involved in development of neuropathic pain and further research investigating the exact role of these key genes is required.
\end{abstract}

Correspondence to: Dr Xiaoping Gu, Department of Anesthesiology, Affiliated Nanjing Drum Tower Hospital of Nanjing University School of Medicine, 321 Zhongshan Road, Nanjing, Jiangsu 210008, P.R. China

E-mail: xiaopinggu@nju.edu.cn

Key words: neuropathic pain, mitogen-activated protein kinase, interleukin-6, transcription factor AP-1, bioinformatics

\section{Introduction}

Neuropathic pain is a chronic pain state associated with multiple etiologies, including trauma, diabetes, cancer, infection and autoimmune pathology (1). The incidence of neuropathic pain is approximately $7-10 \%$ worldwide $(2,3)$. Patients with neuropathic pain induced by nerve injury exhibit varying degrees of mechanical and heat hyperalgesia. Additionally, sleep and anxiety disorders are more prevalent in individuals with neuropathic pain, and quality of life is more seriously impaired, compared with individuals with chronic non-neuropathic pain $(4,5)$. Chronic neuropathic pain also results in considerable social and economic burden, leading to high medical costs and reduced productivity $(6,7)$.

The mechanism underlying neuropathic pain remains unclear and it is still difficult to treat, despite the development of various novel therapies (8). The rapid development of gene chip technology has become a technical basis for biological research; thousands of genes are able to be detected simultaneously with gene chip analysis. At present, large amounts of data have not been effectively explored, despite the availability of large public gene chip databases. Bioinformatics has revealed a considerable number of complex biological interactions through the comprehensive utilization of biology, computer science and statistics (9).

Vega-Avelaira et al (10) used a microarray expression profile to identify differentially expressed genes (DEGs) associated with neuropathic pain. However, the quality of one gene chip studied was not verified. Therefore, for quality control, it should be removed for reanalysis of the normalized unscaled standard errors (NUSE) and RNA degradation curves in the microarray expression profile, as performed by the affy package. The present study reanalyzed the gene expression profile from the Vega-Avelaira et al (10) study to further identify the DEGs associated with neuropathic pain. Function and pathway enrichment analyses of the DEGs were performed, followed by protein-protein interaction (PPI) network construction. Furthermore, reverse transcription-quantitative polymerase chain reaction was performed in a mouse model of neuropathic pain to verify the results obtained from the bioinformatics analysis.

\section{Materials and methods}

Microarray data. The gene expression profile GSE15041 was downloaded from the Gene Expression Omnibus (GEO) 
of the National Center for Biotechnology Information (http://www.ncbi.nlm.nih.gov/gds/) on the Affymetrix Rat Genome 230 2.0 Array (Thermo Fisher Scientific, Inc., Waltham, MA, USA). A total of 9 adult rat samples were analyzed in the present study, including 3 samples from the ipsilateral spinal cord of the sham group (Sham group), 3 samples from the contralateral spinal cord of the surgery group (Contra group) and 3 samples from the ipsilateral spinal cord of the surgery group (Ipsi group). Additionally, the probe annotation package (rat2302.db) of GSE15041 was downloaded from Bioconductor to convert the probe names to gene names (11).

Data preprocessing. Quality control of the gene chip was performed through weighting and residual plot, relative log expression (RLE) box plot,NUSE box plot, principal components plot (PCA) and RNA degradation curve to remove unqualified samples $(12,13)$. The Robust Multi-array Average integrative algorithm was chosen for preprocessing the microarray data, including background correction, quartile data normalization and probe summarization. Following this, the boxplot of the raw and normalized data was drawn (14). The annotate package in $\mathrm{R}$ (15) was subsequently used to convert probe names to gene names. For each sample, a maximum expression value was used for the genes with multiple probe names.

Identification of DEGs. The limma package in R (16) was used to screen out the DEGs (17). Genes with adjusted $\mathrm{P}$ value of $\mathrm{P}<0.05$ and Ifold changel $>2$ were selected as DEGs. Then, cluster analysis and heat map construction was performed using the Pheatmap package (https://cran.r-project. org/package=pheatmap) in R to further analyze the DEGs.

Enrichment analysis of DEGs. Gene function annotation is usually performed by Gene ontology (GO) analysis (18). The GO database consists of three categories, including biological process, molecular function and cellular component. The Kyoto Encyclopedia of Genes and Genomes (KEGG) pathways database is a comprehensive and recognized database encompassing numerous biochemical pathways. In order to gain further insight into the function and pathways of the identified DEGs, the Database for Annotation, Visualization and Integrated Discovery (DAVID; version 6.8) tool (19) was used to perform the GO and KEGG pathway enrichment analysis. The threshold was set as $\mathrm{P}<0.05$ and enrichment counts at $>2$.

PPI network analysis of DEGs. The Search Tool for the Retrieval of Interacting Genes (STRING; version 10.0; http://www.string-db.org/) provides comprehensive and easily accessible interaction information derived from known and predicted protein data (20). Interactions with a combined score of $>0.4$ were selected and a network was built using the STRING online tool. Networks were visualized using Cytoscape software (21) to identify hub proteins and key genes associated with neuropathic pain. Nodes with higher degrees (connected nodes) were considered hub proteins in the PPI network and may have an essential role in the network.

Animals. Male 8-week-old C57BL/6J (weight, 20-23 g; n=10) purchased from Model Animal Research Center of Nanjing
University (Nanjing, China) were used in the present study and were randomly divided into the sham group and surgery group. All animals were housed in a climate-controlled room (temperature: $22 \pm 2^{\circ} \mathrm{C}$; humidity: $50-70 \%$ ) with a $12 \mathrm{~h}$ light/dark cycle for at least 2 weeks, with ad libitum access to food and water. All animal experiments were approved by the Experimental Animals Welfare and Ethical Inspection of Nanjing Drum Tower Hospital (Nanjing, China).

Neuropathic pain model. The most widely used model of neuropathic pain is the unilateral sciatic nerve chronic constriction injury (CCI), developed by Bennet et al (22). Briefly, animals were anesthetized with an intraperitoneal injection of $80 \mathrm{mg} / \mathrm{kg}$ sodium pentobarbital (Sigma-Aldrich; Merck KGaA, Darmstadt, Germany). The skin of the right hind limb was prepared at the mid-thigh level in a lateral position prior to surgery. The sciatic nerve was carefully isolated following skin incision and blunt dissection, two ligatures (5-0 chromic gut suture) were knotted loosely around the sciatic nerve. The distance between ligatures was approximately $1 \mathrm{~mm}$. The surgery was the same in the sham group, however the nerve was only exposed without ligation.

RNA extraction. Mice were deeply anesthetized with sodium pentobarbital and were rapidly decapitated 7 days post-surgery. The lumbar (L4-L5) segments of spinal cords were transversely sectioned and hemi-dissected along the midline. The ipsilateral (CCI-Ipsi) and contralateral (CCI-Contra) lumbar spinal cord of the surgery group and the ipsilateral (Sham-Ipsi) lumbar spinal cord of the sham group was collected. Total RNA was extracted using an RNA extraction kit (Bioteke Corporation, Beijing, China) according to the manufacturer's protocol. RNA concentration was determined using NanoDrop ND-1000 Spectrometer (NanoDrop Technologies; Thermo Fisher Scientific, Inc., Waltham, MA, USA).

$R T-q P C R$. RNA was reverse transcribed into cDNA using Takara PrimeScript RT master mix (RR036A; Takara Biotechnology Co., Ltd., Dalian China). RT-qPCR was performed using an ABI StepOne Plus Real-Time PCR system (Thermo Fisher Scientific, Inc.) and SYBR Premix Ex Taq II master mix (Takara Biotechnology Co., Ltd.) according to the manufacturer's protocol. The reaction system $(10 \mu \mathrm{l})$ consisted of: cDNA $(1 \mu \mathrm{l})$, forward primers $(10 \mu \mathrm{M} ; 0.2 \mu \mathrm{l})$, reverse primers $(10 \mu \mathrm{M} ; 0.2 \mu \mathrm{l}), \mathrm{ROX}$ reference dye $(0.2 \mu \mathrm{l})$, RNase-free water $(3.4 \mu \mathrm{l})$ and SYBR-Green mixture $(5 \mu \mathrm{l})$. The thermocycling conditions were as follows: Initial denaturation, $95^{\circ} \mathrm{C}$ for $30 \mathrm{sec}$, followed by 40 cycles of $95^{\circ} \mathrm{C}$ for $5 \mathrm{sec}$ and $60^{\circ} \mathrm{C}$ for $30 \mathrm{sec}$. GAPDH was used as a housekeeping gene. The relative expression of genes was calculated using the $2^{-\Delta \Delta \mathrm{Ct}}$ method (23). The primer sequences used for RT-qPCR were as follows: GAPDH forward, 5'-TGTGTCCGTCGTGGATCT GA-3' and reverse, 5'-TTGCTGTTGAAGTCGCAGGAG-3'; interleukin (IL)-6 forward, 5'-AGACAAAGCCAGAGTCCT TCA-3' and reverse, 5'-GTGACTCCAGCTTATCTCTTG GT-3'; transcription factor AP-1 (c-Jun) forward, 5'-CCTTCT ACGACGATGCCCTCAA-3' and reverse, 5'-GGGGTCGGT GTAGTGGTGATGT-3'; urikinase-type plasminogen activator (Plau) forward, 5'-AGTGTGGCCAGAAGGCTCTA-3' and reverse, 5'-GCTGCTCCACCTCAAACTTC-3'. 
A

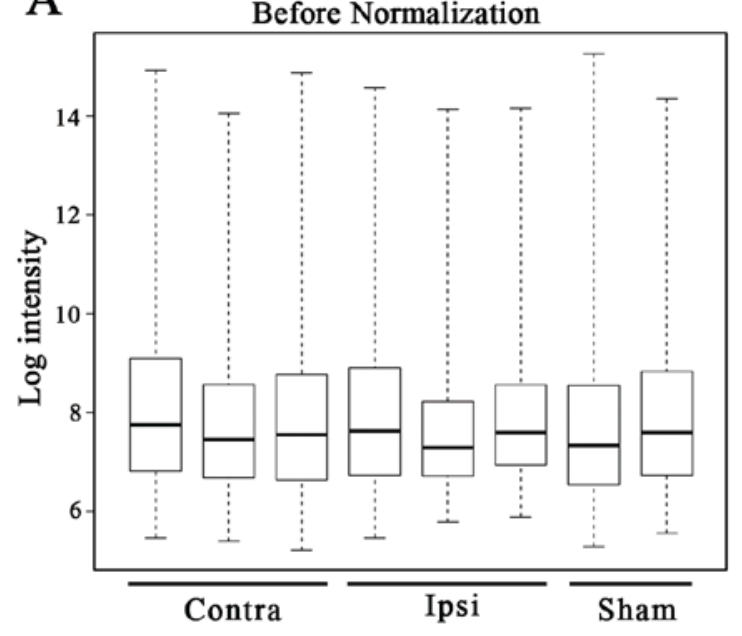

C

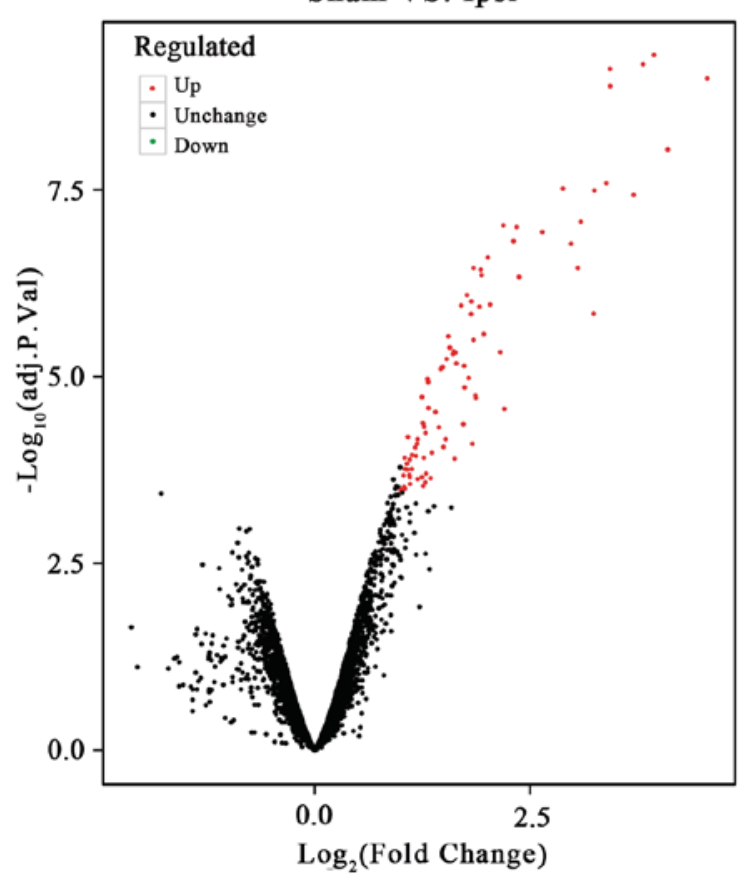

B

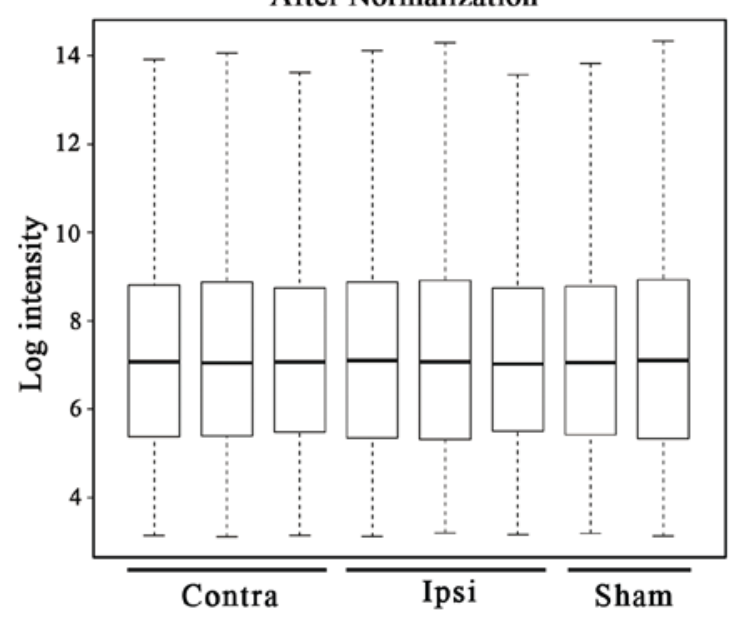

D

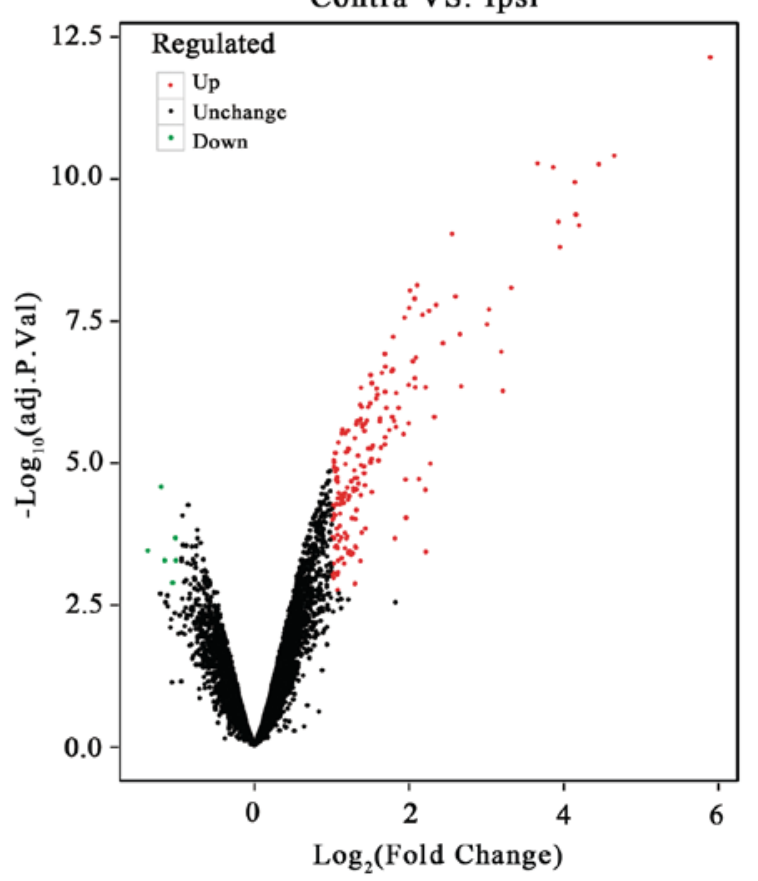

Figure 1. Data preprocessing and DEG screening. Boxplots of log expressed values (A) before and (B) after normalization. (C) Volcanic plot of the DEGs for the Sham group vs. the Ipsi group (D) and the Contra group vs. Ipsi group. Significantly upregulated genes are indicated in red, downregulated in green, and black indicates no significant change. DEG, differentially expressed gene; Sham, ipsilateral spinal cord sham group; Ipsi, ipsilateral spinal cord surgery group; Contra, contralateral spinal cord surgery group.

Statistical analysis. Data are presented as the mean \pm standard deviation. One-way analysis of variance followed by the Bonferroni post hoc test was used to determine the statistical significance, with SPSS software, version 19.0 (IBM Corp., Armonk, NY, USA). P<0.05 was considered to indicate a statistically significant difference.

\section{Results}

Data preprocessing. One disqualified gene chip sample (GSM375686) was removed following quality control of the microarray expression profile. A total of eight samples based on the GPL1355 platform were included for research; this consisted of 2 samples from the Sham group, 3 samples from the Contra group and 3 samples from the Ipsi group. As a result, 13,686 genes were obtained from GSE15041. The box plot of the log expression values for all genes in each sample before and after normalization were drawn (Fig. 1A and B). The median values of each sample were extremely similar, indicating that the data should be further analyzed.

Identification of the DEGs. The limma package in $\mathrm{R}$ was used to perform the DEG analysis. No DEGs were identified between the Sham and Contra group, 90 DEGs were identified between the Sham and Ipsi group and 191 DEGs were identified between the Contra and Ipsi group. The volcanic plot of the Sham vs. Ipsi groups (Fig. 1C) and the Contra vs. Ipsi groups were drawn (Fig. 1D). In total, 86 common DEGs were selected by Venn diagram (Fig. 2A). A heat map of the identified DEGs of the Sham, Contra and Ipsi groups was subsequently constructed 
A

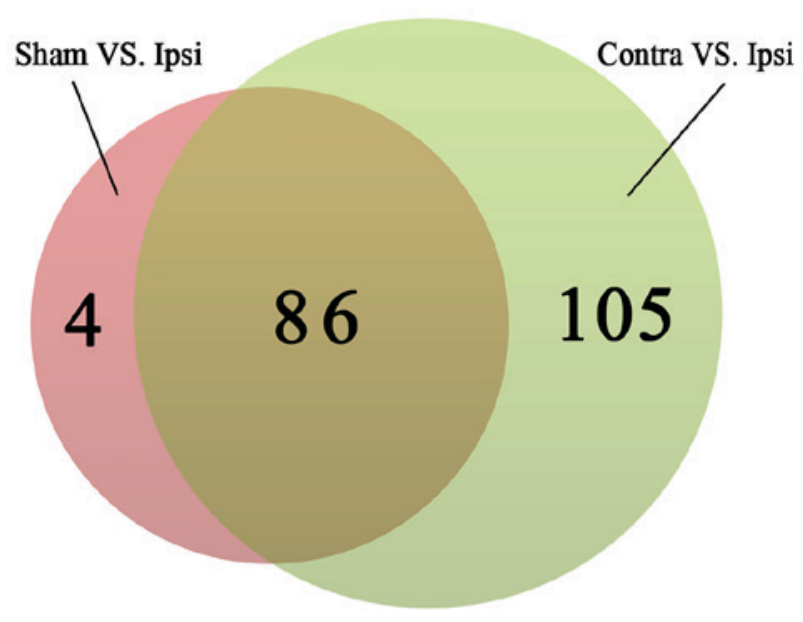

B

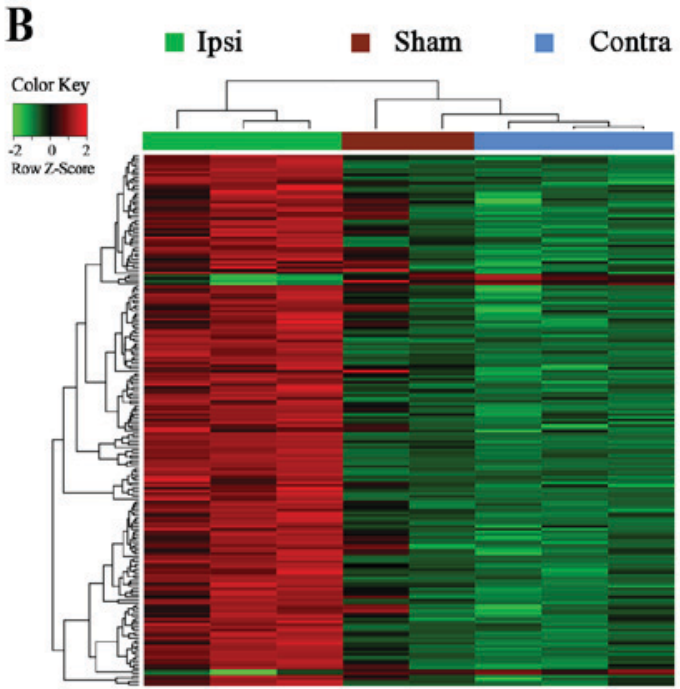

Figure 2. Venn diagram and cluster analysis of identified DEGs. (A) A total of 86 common DEGs were identified from the Venn diagram of the Sham vs. Ipsi (90 DEGs) and Contra vs. Ipsi groups (191 DEGs). (B) Heat map and the clustering pattern of the common DEGs. Red indicates the upregulated genes and green indicates the downregulated genes. Samples were separated into the Ipsi cluster and the Sham and Contra cluster. DEG, differentially expressed gene; Sham, ipsilateral spinal cord sham group; Ipsi, ipsilateral spinal cord surgery group; Contra, contralateral spinal cord surgery group.

to perform cluster analysis (Fig. 2B). The DEG clustering was predominantly separated into two clusters; one cluster was the Ipsi group and the other was the Sham and Contra group.

Function and pathway enrichment analysis. A total of 86 common DEGs were significantly enriched in 77 GO terms (Table I) and 17 KEGG pathways (Table II). Notably, function enrichment analysis revealed that in cellular component terms, DEGs were significantly enriched in 'extracellular space'; in biological processes, DEGs were enriched in response to lipopolysaccharides and cytokines and 'wound healing'; and in molecular function terms, DEGs were enriched in neuropeptide hormone activity. In KEGG pathway enrichment analysis, DEGs were significantly enriched in tuberculosis, rheumatoid arthritis, human T-cell lymphotropic virus type 1 (HTLV-1) and the mitogen-activated protein kinase (MAPK) signaling pathway.

Protein-protein interaction network analysis of DEGs. In the present study, 27 nodes (proteins) and 47 PPI edges (interactions) were obtained following deletion of the disconnected nodes in the network (Fig. 3). IL-6, c-Jun and Plau were considered as hub proteins and key genes (Table III) that may have an important role in the development of neuropathic pain.

$R T-q P C R$ validation. RT-qPCR was used to detect the expression of the identified key genes to confirm the reliability of bioinformatics analysis. Compared with the CCI-Ipsi group, the expression levels of IL-6, c-Jun and Plau were significantly decreased $(\mathrm{P}<0.05)$ in the Sham-Ipsi and CCI-Contra group 7 days post-surgery; no significant difference $(\mathrm{P}>0.05)$ was detected between the Sham-Ipsi group and CCI-Contra group (Fig. 4).

\section{Discussion}

At present, the mechanisms underlying neuropathic pain remain unclear. The development of chronic pain is thought to be due to peripheral and/or central nerve injury. Several studies have reported that nerve injury induces alteration in the peripheral immune system and glial cells (24-26). Additionally, it has been demonstrated that the activation of microglia is associated with the early stages of neuropathic pain in the spinal dorsal horn where the nerve is injured (27-29). Therefore, identifying key genes involved may be helpful in further understanding these mechanisms underlying the development of neuropathic pain.

Vega-Avelaira et al (10) used the microarray expression profile GSE15041 to identify DEGs associated with neuropathic pain. However, the expression profile required re-analysis, as the gene chip quality was not verified. The present study obtained different results following correct quality control. Microarray quality control has a significant impact on the results of the analysis and unverified microarrays may lead to incorrect results. In the present study, mRNA expression was analyzed in a mouse model of neuropathic pain 7 days post-surgery to confirm the bioinformatics analysis results. RT-qPCR was performed following 7 days as mice have the lowest paw withdrawal threshold and this is accompanied by significant changes in molecular biology (30).

A total of 86 common DEGs among the Sham, Contra and Ipsi groups were identified following quality control of the microarray and removal of the disqualified sample. The pivotal roles of IL-6, Jun and Plau in neuropathic pain were subsequently identified.

IL-6 is an inflammatory cytokine with a wide range of biological effects. It has been reported that unilateral sciatic nerve injury increases the expression of IL-6 mRNA (31), and mechanical allodynia induced by spinal nerve lesion is attenuated and delayed in IL-6 knockout mice (32). Furthermore, intrathecal injection of anti-IL-6 antibody reduces mechanical allodynia induced by spinal nerve transection (33). These data indicate the involvement of IL- 6 in the initiation and development of neuropathic pain. Immediate early genes (IEGs) may be transiently and rapidly activated in response 
Table I. Functional enrichment analysis for the common differentially expressed genes.

\begin{tabular}{lllrl}
\hline GO category & GO number & \multicolumn{1}{c}{ GO term } & $\begin{array}{c}\text { Number of differentially } \\
\text { expressed genes }\end{array}$ & P-value \\
\hline CC & GO:0005615 & Extracellular space & 23 & $1.77 \times 10^{-8}$ \\
BP & GO:0071222 & Cellular response to lipopolysaccharide & 8 & $1.02 \times 10^{-5}$ \\
MF & GO:0005184 & Neuropeptide hormone activity & 5 & $1.15 \times 10^{-5}$ \\
BP & GO:0034097 & Response to cytokine & 6 & $8.39 \times 10^{-5}$ \\
BP & GO:0043066 & Negative regulation of apoptotic process & 11 & $1.06 \times 10^{-4}$ \\
BP & GO:0007565 & Female pregnancy & 6 & $1.44 \times 10^{-4}$ \\
BP & GO:0042060 & Wound healing & 6 & $2.61 \times 10^{-4}$ \\
BP & GO:0032496 & Response to lipopolysaccharide & 8 & $2.67 \times 10^{-4}$ \\
BP & GO:0032355 & Response to estradiol & 7 & $2.92 \times 10^{-4}$ \\
BP & GO:0006954 & Inflammatory response & 8 & $3.81 \times 10^{-4}$
\end{tabular}

CC, cellular component; BP, biological process; MF, molecular function; GO, Gene Ontology.

Table II. KEGG pathway enrichment analysis for the common differentially expressed genes.

\begin{tabular}{llcr}
\hline KEGG number & \multicolumn{1}{c}{ KEGG term } & $\begin{array}{c}\text { Number of differentially } \\
\text { expressed genes }\end{array}$ & P-value \\
\hline rno05152 & Tuberculosis & 8 & $2.79 \times 10^{-5}$ \\
rno05323 & Rheumatoid arthritis & 5 & $1.01 \times 10^{-3}$ \\
rno04145 & Phagosome & 6 & $2.77 \times 10^{-3}$ \\
rno05166 & Human T-cell lymphotropic virus type 1 infection & 7 & $2.99 \times 10^{-3}$ \\
rno05321 & Inflammatory bowel disease & 4 & $4.10 \times 10^{-3}$ \\
rno05202 & Transcriptional misregulation in cancer & 5 & $8.45 \times 10^{-3}$ \\
rno04010 & Mitogen-activated protein kinase signaling pathway & 6 & $8.98 \times 10^{-3}$ \\
rno04514 & Cell adhesion molecules & 5 & $1.04 \times 10^{-2}$ \\
rno04612 & Antigen processing and presentation & 4 & $1.24 \times 10^{-2}$ \\
rno05146 & Amoebiasis & 4 & $1.82 \times 10^{-2}$ \\
\hline
\end{tabular}

KEGG, Kyoto Encyclopedia of Genes and Genomes.

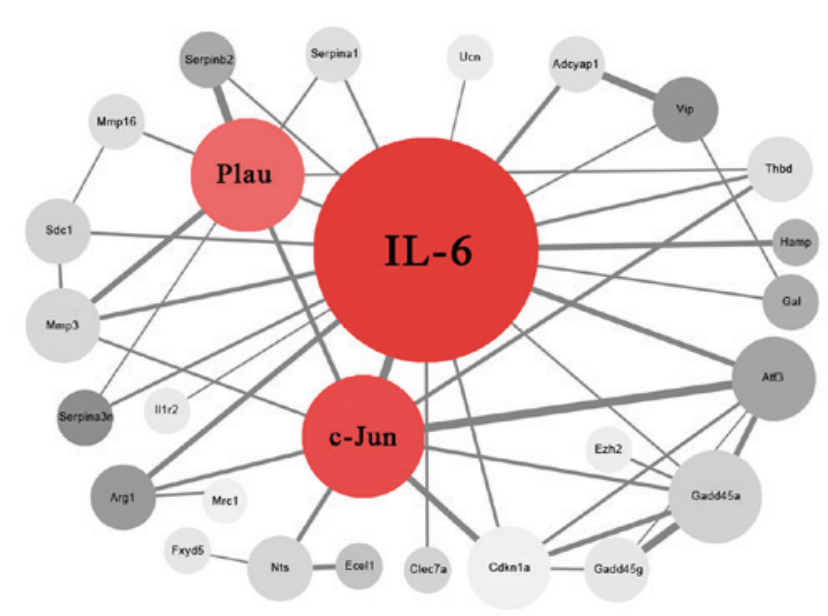

Figure 3. Protein-protein interaction network of the identified differentially expressed genes. The degree of color indicates the degree of fold change (lighter grey indicate a lower fold change and darker grey a higher fold change) and the size of circle represents the node degree. IL-6, c-Jun and Plau were identified as key genes. IL-6, interleukin-6; c-Jun, transcription factor AP-; Plau, urikinase-type plasminogen activator. to a wide variety of cellular stimuli. Well-characterized IEGs include c-Jun, and the proto-oncogenes c-Fos and c-Myc. Double labeling with the tracers fast blue and horseradish peroxidase-labeled gold underwent retrograde transport from the site of injury and revealed that c-Jun protein expression was confined to injured neurons (34). Activated c-Jun in the dorsal root ganglion (DRG) contributes to the pathogenesis of neuropathic pain induced by CCI (35), and neuropathic pain is alleviated by inhibiting the mitogen-activated protein kinase 8 (JNK)/c-Jun signal pathway $(36,37)$. Furthermore, Plau mRNA is abundantly expressed by many neurons in the peripheral and central nervous system (38). Plau expression is upregulated in rat DRGs following nerve injury (39). The aforementioned data suggests the involvement of IL-6, c-Jun and Plau in neuropathic pain.

Common DEGs were revealed to be enriched in the injury and inflammation-associated biological processes through GO enrichment analysis. Pathway analysis revealed that DEGs were typically enriched in tuberculosis, rheumatoid arthritis, HTLV-1 and MAPK signaling pathways. Spinal tuberculosis 
Table III. Hub proteins in the protein-protein interaction network.

\begin{tabular}{lc}
\hline Proteins & Degree \\
\hline IL-6 & 20 \\
Jun & 9 \\
Plau & 8 \\
Gadd45 $\alpha$ & 6 \\
Atf3 & 5 \\
Cdkn1a & 5
\end{tabular}

IL-6, interleukin 6; c-Jun, transcription factor AP-1; Plau, urikinase-type plasminogen activator; Gadd $45 \alpha$, growth arrest and DNA damage-inducible protein GADD45 $\alpha$; Atf3, cyclic AMP-dependent transcription factor Atf3; Cdkn1a, cyclin-dependent kinase inhibitor 1.

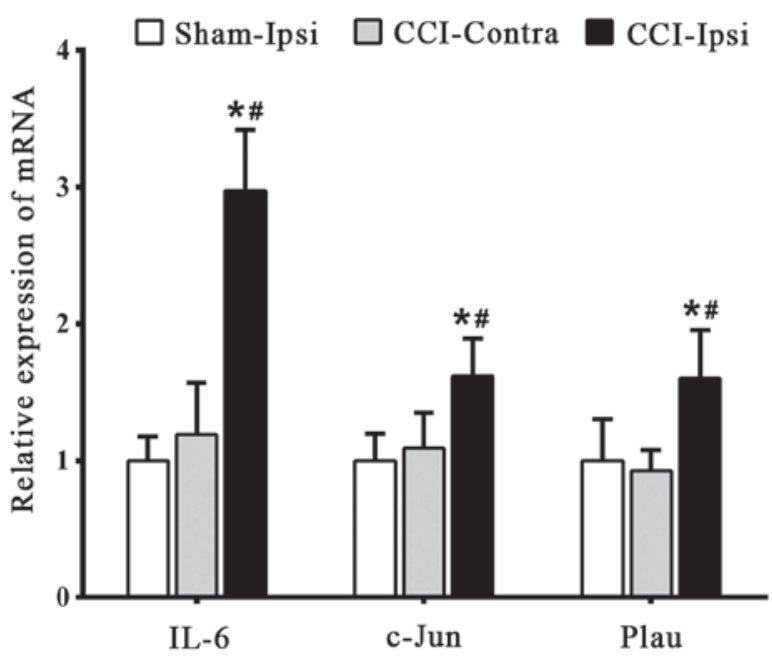

Figure 4. Expression of key genes in a mouse model of neuropathic pain. Reverse transcription-quantitative polymerase chain reaction was performed to detect the mRNA expression of IL-6, c-Jun and Plau. * $\mathrm{P}<0.05$ vs. Sham-Ipsi group, ${ }^{\#} \mathrm{P}<0.05$ vs. CCI-Contra group. IL-6, interleukin 6; c-Jun, transcription factor AP-1; Plau, urikinase-type plasminogen activator; Sham, sham group; Ipsi, ipsilateral lumbar spinal cord; Contra, contralateral lumbar spinal cord; CCI, chronic constriction injury.

may induce varying degrees of damage to the spinal cord nerve roots (40), resulting in neuropathic pain. A clinical study revealed that a third of patients with rheumatoid arthritis have symptoms of neuropathic pain (41), and a review reported that approximately $53.1 \%$ of HTLV-1 carriers have symptoms of neuropathic pain (42). The conventional MAPKs include the p38 isoforms, extracellular signal-regulated kinases $1 / 2$ (ERK1/2) and JNKs (43). MAPKs regulate numerous physiological activities, including inflammation, apoptosis, cancer, tumor cell invasion and metastasis. Active MAPK signaling also has an important role in neuropathic pain. It has been reported that IL-6 is involved in the maintenance of neuropathic pain by activating ERK; and the JNK/c-Jun pathway also participates in the maintenance of neuropathic pain (43). It has been demonstrated that p38 MAPK activation in the ipsilateral spinal dorsal horn is significantly increased following CCI (44). Additionally, MAPK activity is significantly decreased following an intrathecal injection of anti-IL-6 antibody (45). These results indicate that IL-6 and c-Jun have an important role in neuropathic pain that is mediated through the MAPK signaling pathway. This is consistent with the bioinformatics findings of the present study and reflect the reliability of the bioinformatics analysis performed.

In the present study, the results of the bioinformatics analysis revealed that there was a significant difference between the Sham and Ipsi group $(\mathrm{P}<0.05)$, however no difference was observed between the Sham and Contra group ( $\mathrm{P}>0.05)$. RT-qPCR analysis was consistent with this. The sciatic nerve was only injured in the CCI-Ipsi group, not the CCI-Contra or Sham-Ipsi group, and the nerve was only exposed in the Sham-Ipsi group. Sciatic nerve injury induces the inflammatory response in the ipsilateral spinal cord, whereas the contralateral spinal cord was not, compared with the sham group, as previously reported (46). Nerve injury induces a set of progressive alterations in ipsilateral spinal cord microglia with increased secretion of cytokines. This does not occur in the contralateral spinal cord following nerve injury, or the uninjured ipsilateral spinal cord. Therefore, significant changes in neurophysiology in spinal cord of the Sham and Contra group may not be induced. DEG analysis revealed that there were numerous DEGs detected in the Ipsi group compared with the Contra and Sham groups, and not between the Contra and Sham groups. This may be due to only the Ipsi group exhibiting nerve injury, whereas the other two groups did not. However, DEGs may be detected between the Contra and Sham group only in the local tissue of the surgical incision, rather than the spinal cord. Thus, many DEGs were identified in the Ipsi group compared with Sham and Contra group by bioinformatics analysis.

In summary, 86 DEGs were identified and IL-6, c-Jun, Plau were identified as key genes in neuropathic pain through pathway and PPI network analysis, which may aid in further understanding of the underlying pain mechanisms. Following the bioinformatics analysis, the expression of key genes was confirmed with RT-qPCR in a mouse model of neuropathic pain. However, the current study has certain limitations. Although the results revealed that IL-6, c-Jun and Plau have an important role in neuropathic pain, further studies are required to confirm the results at the molecular level.

\section{Acknowledgements}

The authors of the present study would like to thank their colleagues for their support and help.

\section{Funding}

The present study supported by the National Natural Science Foundation of China (grant nos. 81471129, 81371207, 81171047 and 81171048), the Natural Science Foundation of Jiangsu Province of China (grant no. BK20170654), the Department of Health of Jiangsu Province of China (grant nos. XK201140 and RC2011006) and the Fundamental Research Funds for the Central Universities (grant nos. 0214-14380338 and 0214-14380271). 


\section{Availability of data and materials}

The analyzed datasets generated during the study are available from the corresponding author on reasonable request.

\section{Author's contributions}

Study Design: HL, TX, ZM and XG; data collection: HL; statistical analysis: HL and TX; animal experiment: FX and HL; manuscript preparation: HL, TX and XG; funds collection: TX, ZM and XG.

\section{Ethics approval and consent to participate}

All animal experiments were approved by the Experimental Animals Welfare and Ethical Inspection of Nanjing Drum Tower Hospital (Nanjing, China).

\section{Consent for publication}

Not applicable.

\section{Competing interests}

The authors declare that they have no competing interests.

\section{References}

1. Woolf CJ and Mannion RJ: Neuropathic pain: Aetiology, symptoms, mechanisms, and management. Lancet 353: 1959-1964, 1999.

2. van Hecke O, Austin SK, Khan RA, Smith BH and Torrance N: Neuropathic pain in the general population: A systematic review of epidemiological studies. Pain 155: 654-662, 2014.

3. Bouhassira D, Lanteri-Minet M, Attal N, Laurent B and Touboul C: Prevalence of chronic pain with neuropathic characteristics in the general population. Pain 136: 380-387, 2008.

4. Attal N, Lanteri-Minet M, Laurent B, Fermanian J and Bouhassira D: The specific disease burden of neuropathic pain: Results of a French nationwide survey. Pain 152: 2836-2843, 2011.

5. Finnerup NB, Haroutounian S, Kamerman P, Baron R, Bennett DL, Bouhassira D, Cruccu G, Freeman R, Hansson P, Nurmikko T, et al: Neuropathic pain: An updated grading system for research and clinical practice. Pain 157: 1599-1606, 2016.

6. Attal N and Bouhassira D: Pharmacotherapy of neuropathic pain: Which drugs, which treatment algorithms? Pain 156 (Suppl 1): S104-S114, 2015

7. Gewandter JS, Dworkin RH, Turk DC, Farrar JT, Fillingim RB, Gilron I, Markman JD, Oaklander AL, Polydefkis MJ, Raja SN, et al: Research design considerations for chronic pain prevention clinical trials: IMMPACT recommendations. Pain 156: 1184-1197, 2015.

8. Finnerup NB, Sindrup SH and Jensen TS: Recent advances in pharmacological treatment of neuropathic pain. F1000 Med Rep 2: 52, 2010.

9. Luscombe NM, Greenbaum D and Gerstein M: What is bioinformatics? A proposed definition and overview of the field. Methods Inf Med 40: 346-358, 2001

10. Vega-Avelaira D, Géranton SM and Fitzgerald M: Differential regulation of immune responses and macrophage/neuron interactions in the dorsal root ganglion in young and adult rats following nerve injury. Mol Pain 5: 70, 2009.

11. Carlson M: Rat2302.db: Affymetrix Rat Genome 2302.0 Array annotation data (chip rat2302). R package version 3.2.3, 2016.

12. Gautier L, Cope L, Bolstad BM and Irizarry RA: Affy--analysis of Affymetrix GeneChip data at the probe level. Bioinformatics 20: 307-315, 2004.

13. Wilson CL and Miller CJ: Simpleaffy: A BioConductor package for Affymetrix quality control and data analysis. Bioinformatics 21: 3683-3685, 2005.
14. Irizarry RA, Hobbs B, Collin F, Beazer-Barclay YD, Antonellis KJ, Scherf U and Speed TP: Exploration, normalization, and summaries of high density oligonucleotide array probe level data. Biostatistics 4: 249-264, 2003.

15. Gentleman R: Annotate: Annotation for microarrays. R package version 1.56.1, 2017.

16. Ritchie ME, Phipson B, Wu D, Hu Y, Law CW, Shi W and Smyth GK: 'Limma powers differential expression analyses for RNA-sequencing and microarray studies.' Nucleic Acids Res 43: e47, 2015 .

17. Smyth GK: Linear models and empirical bayes methods for assessing differential expression in microarray experiments. Stat Appl Genet Mol Biol 3: Article3, 2004.

18. Ashburner M, Ball CA, Blake JA, Botstein D, Butler $\mathrm{H}$, Cherry JM, Davis AP, Dolinski K, Dwight SS, Eppig JT, et al: Gene ontology: Tool for the unification of biology. The Gene Ontology Consortium. Nat Genet 25: 25-29, 2000.

19. Huang da W, Sherman BT and Lempicki RA: Systematic and integrative analysis of large gene lists using DAVID bioinformatics resources. Nat Protoc 4: 44-57, 2009.

20. Szklarczyk D, Franceschini A, Kuhn M, Simonovic M, Roth A, Minguez P, Doerks T, Stark M, Muller J, Bork P, et al: The STRING database in 2011: Functional interaction networks of proteins, globally integrated and scored. Nucleic Acids Res 39: D561-D568, 2011.

21. Smoot ME, Ono K, Ruscheinski J, Wang PL and Ideker T: Cytoscape 2.8: New features for data integration and network visualization. Bioinformatics 27: 431-432, 2011.

22. Bennett GJ and Xie YK: A peripheral mononeuropathy in rat that produces disorders of pain sensation like those seen in man. Pain 33: 87-107, 1988

23. Livak KJ and Schmittgen TD: Analysis of relative gene expression data using real-time quantitative PCR and the 2(-Delta Delta C(T)) method. Methods 25: 402-408, 2001

24. Scholz J and Woolf CJ: The neuropathic pain triad: Neurons, immune cells and glia. Nat Neurosci 10: 1361-1368, 2007.

25. Milligan ED and Watkins LR: Pathological and protective roles of glia in chronic pain. Nat Rev Neurosci 10: 23-36, 2009.

26. Thacker MA, Clark AK, Marchand F and McMahon SB: Pathophysiology of peripheral neuropathic pain: Immune cells and molecules. Anesth Analg 105: 838-847, 2007.

27. Inoue $\mathrm{K}$ and Tsuda M: Microglia and neuropathic pain. Glia 57: 1469-1479, 2009.

28. Dominguez E, Rivat C, Pommier B, Mauborgne A and Pohl M: JAK/STAT3 pathway is activated in spinal cord microglia after peripheral nerve injury and contributes to neuropathic pain development in rat. J Neurochem 107: 50-60, 2008.

29. Tsuda M, Shigemoto-Mogami Y, Koizumi S, Mizokoshi A Kohsaka S, Salter MW and Inoue K: P2X4 receptors induced in spinal microglia gate tactile allodynia after nerve injury. Nature 424: 778-783, 2003.

30. Lai CY,Hsieh MC, Ho YC, Lee AS, Wang HH, Cheng JK, Chau YP and Peng HY: Growth Arrest and DNA-damage-inducible protein $45 \beta$-mediated DNA demethylation of voltage-dependent t-type calcium channel 3.2 subunit enhances neuropathic allodynia after nerve injury in rats. Anesthesiology 126: 1077-1095, 2017.

31. Luo X, Tai WL, Sun L, Pan Z, Xia Z, Chung SK and Cheung CW: Crosstalk between astrocytic CXCL12 and microglial CXCR4 contributes to the development of neuropathic pain. Mol Pain 12, 2016.

32. Ramer MS, Murphy PG, Richardson PM and Bisby MA: Spinal nerve lesion-induced mechanoallodynia and adrenergic sprouting in sensory ganglia are attenuated in interleukin- 6 knockout mice. Pain 78: 115-121, 1998.

33. Arruda JL, Sweitzer S, Rutkowski MD and DeLeo JA: Intrathecal anti-IL-6 antibody and IgG attenuates peripheral nerve injury-induced mechanical allodynia in the rat: Possible immune modulation in neuropathic pain. Brain Res 879: 216-225, 2000.

34. Leah JD, Herdegen T and Bravo R: Selective expression of Jun proteins following axotomy and axonal transport block in peripheral nerves in the rat: Evidence for a role in the regeneration process. Brain Res 566: 198-207, 1991.

35. Son SJ, Lee KM, Jeon SM, Park ES, Park KM and Cho HJ: Activation of transcription factor c-jun in dorsal root ganglia induces VIP and NPY upregulation and contributes to the pathogenesis of neuropathic pain. Exp Neurol 204: 467-472, 2007.

36. Wang C, Kong X, Zhu C, Liu C, Sun D, Xu Q, Mao Z, Qin Q, $\mathrm{Su} \mathrm{H}$, Wang D, et al: Wu-tou decoction attenuates neuropathic pain via suppressing spinal astrocytic IL-1R1/TRAF6/JNK signaling. Oncotarget 8: 92864-92879, 2017. 
37. Jiang L, Pan CL, Wang CY, Liu BQ, Han Y, Hu L, Liu L, Yang Y, Qu JW and Liu WT: Selective suppression of the JNK-MMP2/9 signal pathway by tetramethylpyrazine attenuates neuropathic pain in rats. J Neuroinflammation 14: 174, 2017.

38. Sumi Y, Dent MA, Owen DE, Seeley PJ and Morris RJ: The expression of tissue and urokinase-type plasminogen activators in neural development suggests different modes of proteolytic involvement in neuronal growth. Development 116: 625-637, 1992.

39. Yamanaka H,Obata K, Fukuoka T,Dai Y,Kobayashi K, Tokunaga A and Noguchi K: Tissue plasminogen activator in primary afferents induces dorsal horn excitability and pain response after peripheral nerve injury. Eur J Neurosci 19: 93-102, 2004.

40. Wouda EMN, Stienstra Y, van der Werf TS, Kerstjens H, de Lange WCM, Coppes M, Kuijlen J, Tepper M and Akkerman OW: Neurological and functional recovery in tuberculosis patients with spinal cord injury in the Netherlands. NeuroRehabilitation 40: 439-445, 2017.

41. Yesil H, Sungur U, Akdeniz S, Gurer G, Yalcin B and Dundar U: Association between serum vitamin D levels and neuropathic pain in rheumatoid arthritis patients: A cross-sectional study. Int J Rheum Dis 21: 431-439, 2018.

42. San-Martin DL, Santos DN and Baptista AF; Pain Study Group: Pain prevalence, characteristics and associated factors in human T-cell lymphotropic virus type 1 infected patients: A systematic review of the literature. Braz J Infect Dis 20: 592-598, 2016.
43. Ding CP, Guo YJ, Li HN, Wang JY and Zeng XY: Red nucleus interleukin-6 participates in the maintenance of neuropathic pain through JAK/STAT3 and ERK signaling pathways. Exp Neurol 300: 212-221, 2018.

44. Zhao WX, Wang PF, Song HG and Sun N: Diosgenin attenuates neuropathic pain in a rat model of chronic constriction injury. Mol Med Rep 16: 1559-1564, 2017.

45. Zaringhalam J, Tekieh E, Manaheji H and Akhtari Z: Cellular events during arthritis-induced hyperalgesia are mediated by interleukin- 6 and p38 MAPK and their effects on the expression of spinal mu-opioid receptors. Rheumatol Int 33: 2291-2299, 2013.

46. Banno T, Omura T, Masaki N, Arima H, Xu D, Okamoto A, Costigan M, Latremoliere A, Matsuyama Y and Setou M: Arachidonic acid containing phosphatidylcholine increases due to microglial activation in ipsilateral spinal dorsal horn following spared sciatic nerve injury. PLoS One 12: e0177595, 2017.

This work is licensed under a Creative Commons Attribution-NonCommercial-NoDerivatives 4.0 International (CC BY-NC-ND 4.0) License. 